\title{
Salicylic acid peel in pigmented skin
}

Keywords: salicylic acid, acne vulgaris, fitzpatrick types. Desmosomes, sebum

\section{Opinion}

Salicylic acid is a beta hydroxyl acid that has the capacity to destroy the superficial layer of the skin. It had a desmolytic effect; being able to destroy the protein components of the desmosomes which are special structures that holds the keratinocytes together in the epidermis. This leads to the exfoliation of the epidermis. At various concentrations, the effect of Salicylic acid varies in the epidermis with extension to the upper dermis.

Salicylic acid peel is one of the numerous peels used in the treatment of acne and control of excessive sebum secretion. Various studies had reported the effectiveness of Salicylic acid (20\%-30\%) peel in the management of acne vulgaris. However, there are reports of transient pigmentary changes at such concentrations in patients with Fitzpatrick types IV-VI.

In my opinion, Salicylic acid at concentrations of $15-20 \%$ remains the best option for superficial peel in pigmented skin (Fitzpatrick types $\mathrm{V}$ and VI) with acne and/or excessive sebum secretion. At a lower concentration (15\%-20\%), Salicylic acid peel is equally effective in pigmented skin with acne. It reduces the number of both inflammatory and non-inflammatory lesions. It has a comedolytic effect because of the exfoliation of the epidermis. In addition, it has anti-inflammatory effects, reducing the effects of inflammatory cytokines.

Salicylic acid is lipophilic; therefore, it can penetrate to the sebaceous glands when applied to the epidermis. It reduces secretion of sebum causing dryness of the facial skin. This is beneficial in reducing comedone formation and controlling sebum secretion in patients with excessive secretion of sebum. However, in the few acne patients with normal or low sebum secretion, lower concentration $(15 \%)$ of Salicylic acid peels will be effective with minimal discomfort.

\author{
Volume 2 Issue I - 2018

\section{Emeka O Okoro} \\ Department of Medicine, Federal Medical Centre, Nigeria
}

Correspondence: Emeka O Okoro, Dermatology unit, Department of Medicine, Federal Medical Centre, Keffi, Nasarawa State, Nigeria, Tel +2348I38353294, Emailemybum@yahoo.com

Received: February II, 2018 | Published: February 16, 2018

One of the common complications of chemical peels in pigmented skin types is the risk of dyschromia especially post-inflammatory hyper pigmentation. There is little or no risk of post-inflammatory hyper pigmentation when using Salicylic acid peels in pigmented skin. This is unlike the use of Trichloroacetic acid peel in pigmented skin where post-inflammatory hyper pigmentation can occur.

Salicylic acid peel is cheap and affordable. This makes it an ideal peel in resource poor setting and developing African nations. It is readily available and effective for patients who may not afford the more expensive options.

In conclusion, superficial chemical peels are important in the treatment of acne in skin types IV-VI. Salicylic acid peels at concentrations of $15 \%-20 \%$ is the better option for pigmented skin types with acne and excessive sebum secretion especially in resource poor setting.

\section{Acknowledgements}

None.

\section{Conflicts of interest}

The author declares no conflict of interest. 